\title{
ТЕНЗОРНОЕ ВЫРАЖЕНИЕ ДЛЯ ГИПОТЕЗЫ БУССИНЕСКА
}

\author{
С. П. Сергеев, С. В. Афанасьев, Ю.Н. Шевченко
}

СТАНИСЛАВ ПЕТРОВИЧ СЕРГЕЕВ - ОАО «Научно-исследовательский и проектньий институт азотной промышленности и продуктов органического синтеза».E-mail: stanislav.sergeev@giap-m.com

СЕРГЕЙ ВАСИЛЬЕВИЧ АФАНАСЬЕВ - ПАО «ТОАЗ».E-mail: svaf77@mail.ru.

ЮЛИЯ НИКОЛАЕВНА ШЕВЧЕНКО - Кафедра «Химическая технология и ресурсосбережение», Тольяттинский государственный университет.E-mail: jnshevchenko@gmail.com.

ОАО «Научно-исследовательский и проектный институт азотной промылиленности и продуктов органического синтеза», ул. Земляной Вал, 50A/8, стр. 4, Москва, Российская Федераџия, 109028.

ПАО «ТОАЗ», 445045, Самарская область, г. Тольятти, Поволжское шоссе, 32.

Тольяттинский государственный университет., ул. Белорусская, 14, г. Тольятти, Российская Федерация, 445020

В статье приведен краткий обзор использования понятия «кажущейся турбулентной вязкости» для описания турбулентного течения жидкости или газа в каналах.

Рассмотрены теоретические основы математического описания параметра турбулентной вязкости в уравнении Рейнольдса в тензорной форме. Для количественной интерпретации процессов, связанных с явлением турбулентности, например, переносом массы и импульса, применён аппарат статистической физики и механики.

На конкретных примерах показано, что использование понятия «кажущейся турбулентной вязкости» в скалярной форме для описания двух- или трехмерного течения вязкой жидкости, возникающего, например, в каналах с проницаемыли стенками, лишено физического смысла.

В ходе проведённых исследований впервые подтверждено, что математические соотношения для интегральных параметров течения, базирующиеся на значениях пульсаџий скорости и её приращения можно выразить через пульсацию координат и их приращений. Одновременно с этим выявлено, что пульсацุия приращцения координат, равна пульсащчи координат, а пульсащчя приращений лнейной скорости равна пульсации скорости. Подтверждена коллинеарность пульсаџий векторов длинь (амплитуды пульсаций) и скорости.

В ходе анализа математических выражений установлено, что выражения для коэффициентов турбулентной вязкости и диффузии можно рассматривать в качестве математического тензора.

Результатом проведенных исследований является полученное авторами тензорное выражение для коэффициента турбулентной вязкости и турбулентной диффузии. Выписань уравнения для определения среднеквадратичных пульсаций компонент вектора скорости $-\left(\overline{V_{1}^{\prime 2}}\right),-\left(\overline{V_{2}^{\prime 2}}\right),-\left(\overline{V_{1}^{\prime} V_{2}^{\prime}}\right)$ для течения в плоском прямоугольном канале, вытекающие из результирующего выражения для тензора турбулентных напряжений в несжимаемой жидкости.

Показано качественное соответствие выражений для компонент тензора турбулентных напряжений для двухмерного плоского течения с известными экспериментальными данными, установленными при исследовании течения воздуха в плоском прямоугольном канале.

Ключевые слова: турбулентность, течения с отсосом и вдувом, турбулентная вязкость. 


\title{
TENSOR EXPRESSION FOR A HYPOTHESIS OF BUSSINESK
}

\author{
S.P. Sergeev ${ }^{1}$, S. V. Afanasiev ${ }^{2}$, J. N. Shevchenko ${ }^{3}$ \\ ${ }^{1}$ OJSC "Scientific-Research and Design Institute of Nitrogen Industry and Organic Synthesis Products", Zemlyanoy \\ val st., 50A/8, p. 4, Moscow, 109028, Russia.
}

${ }^{2} P A O$ "TOAZ”, 445045, Samara region, Tolyatti, Volga highway, 32.

${ }^{3}$ Department of "Environmental Management and Resource saving”, Togliatti State University, Belorusskaya st., 14, Togliatti, 445020, Russia.

The article provides a brief overview of the use of the concept of "apparent turbulent viscosity" to describe the turbulent flow of a liquid or gas in channels.

The theoretical foundations of the mathematical description of the turbulent viscosity parameter in the Reynolds equation in tensor form are considered. For a quantitative interpretation of the processes associated with the phenomenon of turbulence, for example, mass and momentum transfer, the apparatus of statistical physics and mechanics is used.

Using specific examples, it is shown that the use of the concept of "apparent turbulent viscosity" in scalar form to describe a two- or three-dimensional flow of a viscous fluid that occurs, for example, in channels with permeable walls, is devoid of physical meaning.

In the course of the research, it was first confirmed that the mathematical relations for the integral parameters of the flow, based on the values of the velocity pulsations and its increments, can be expressed through the pulsation of coordinates and their increments. At the same time, it was revealed that the ripple of the increment of coordinates is equal to the ripple of coordinates, and the ripple of increments of linear velocity is equal to the ripple of speed. The collinearity of the pulsations of the vectors of length (pulsation amplitude) and velocity is confirmed.

In the analysis of mathematical expressions, it was found that the expressions for the coefficients of turbulent viscosity and diffusion can be considered as a mathematical tensor.

The result of the research is the tensor expression obtained by the authors for the coefficient of turbulent viscosity and turbulent diffusion. Equations are written for determining the rms pulsations of the components

of the velocity vector $-\left(\overline{V_{1}^{\prime 2}}\right),-\left(\overline{V_{2}^{\prime 2}}\right),-\left(\overline{V_{1}^{\prime} V_{2}^{\prime}}\right)$ for flow in a flat rectangular channel, resulting from the resulting expression for the tensor of turbulent stresses in an incompressible fluid.

The qualitative correspondence of the expressions for the components of the turbulent stress tensor for a twodimensional plane flow with the known experimental data established in the study of air flow in a rectangular rectangular channel is shown.

Keywords: turbulence, currents with a suction and blow in, turbulent viscosity.

\section{Введение}

Турбулентные течения наиболее распространенный вид течений в трубопроводах, структурных элементах химико-технологических установок или в высокоскоростных струях. Для моделирования подобных течений используется устоявшееся понятие кажущейся кинематической вязкости, коэффициенты которой определяются экспериментально или по известным физическим моделям. Большинство моделей используют «обоб- щенную (с добавкой, учитывающей сжимаемость среды)» гипотезу Буссинеска или представление Прандтля [1-11].

Для описания турбулентных течений обычно исходят из уравнений Рейнольдса, в которых действительное давление и действительная скорость течения выражаются через сумму осредненных значений и пульсационных добавок к ним. С учетом этого уравнение сохранения импульса, записанное для осредненных по времени значений переменных в тензорной форме [12-15], имеет вид [16]: 
$\rho\left(\frac{\partial V_{j}}{\partial t}+\frac{\partial V_{j} V_{k}}{\partial x_{j} \partial x_{k}}\right)=\frac{\partial}{\partial x_{k}}\left(-P \delta_{j k}+\tau_{j k}-\rho \overline{V_{j}^{\prime} V_{k}^{\prime}}\right)$

Тензор вязких напряжений:

$$
\text { Здесь } \tau_{j k}=v \rho S_{j k}=v \rho\left(\frac{\partial V_{j}}{\partial x_{k}}+\frac{\partial V_{k}}{\partial x_{j}}\right)
$$

Проблемой при использовании уравнения (1) является представление пульсационных напряжений, выражаемых тензором в подходящей форме, пригодной для дальнейших вычислений:

$$
-\rho \overline{V_{j}^{\prime} V_{k}^{\prime}}=T_{j k}
$$

Эта непростая задача и в настоящее время вызывает большой интерес исследователей ввиду распространенности турбулентных течений и их важности для технических приложений и остается актуальной.

«Обобщённая» гипотеза Буссинеска основывается на аналогии с законом вязкости Ньютона. В обозначениях, принятых в этой статье для несжимаемой жидкости она имеет вид:

$$
T_{j k}=\rho \varepsilon S_{j k}
$$

В этом выражении величина коэффициента кажущейся кинематической вязкости $\varepsilon$ является скаляром. Помимо того, что коэффициент $\varepsilon$ зависит от скорости и координат, формула (4), строго говоря, не имеет физического смысла, так как след тензора $\mathrm{S}_{\mathrm{jk}}$, представляющий уравнение неразрывности равняется нулю.

$$
\operatorname{tr} T_{j k}=\rho \varepsilon\left(t r S_{j k}\right)=0
$$

Однако левая часть уравнения (5) может равняться нулю в соответствии с уравнением (3) только при полном отсутствии турбулентности. На это принципиальное обстоятельство указал Слеттери [17].

Тем не менее гипотеза Буссинеска послужила основой для создания других гипотез, суть которых заключалась в нахождении зависимости коэффициента кажущейся турбулентной вязкости от поля скорости и координат. Важнейший вклад в решение этой задачи был внесен Л. Прандтлем [18]. Модель Прандтля получила название теории «пути смешения». В своем самом простом виде она приводила к следующему выражению для турбулентных напряжений в плоском двумерном потоке:

$$
\tau_{t}=\rho l^{2}\left|\frac{d \bar{u}}{d y}\right| \cdot \frac{d \bar{u}}{d y}
$$

Похожую формулу, отличающуюся от (6) только коэффициентом 1/2 получил Дж. Тейлор из теории переноса завихренности [19].

Теории Прандтля, Тейлора, Кармана использовались для получения универсальных законов распределения скоростей и сопротивлений для течений в трубах и плоских каналах и явились значительными достижениями в гидродинамике [19].

Получение более общих тензорных выражений для турбулентных напряжений (из формулы (1) видно, что турбулентные напряжения - математический тензор) является необходимым для решения некоторых важных технических задач. К ним относятся, например, задачи расчета течений в каналах с сильным отсосом или вдувом. Такие течения часто встречаются в радиальных каталитических реакторах [20], адсорберах, коллекторах и распределителях змеевиковых теплообменников [21] и других аппаратах химической технологии.

Для ламинарного или осесимметричного течения уравнение Навье-Стокса может быть сведено к уравнению в частных производных для одной переменной в виде функции течения

$$
F(u, \vartheta)=\frac{1}{\bar{V}_{x}} \int_{0}^{\vartheta} V_{x} d \vartheta
$$

Решение этого уравнения принесло весьма плодотворные результаты [22]. Однако, такое уравнение не может быть получено для турбулентного потока, пока не известно тензорное выражение для турбулентной вязкости. В связи с этим, насколько известно, подобные попытки и не предпринимались. Ниже рассматривается возможный путь получения тензорного выражения для гипотезы Буссинеска.

\section{Методика эксперимента}

Предположим, что в турбулентном потоке измеряется средняя скорость жидкости $V_{j}$ и её пульсации $V_{j}^{\prime}$ в точке с координатами $\left(x_{1}, x_{2}, x_{3}\right)$. Если известна средняя по времени скорость жидкости $V_{j}^{0}$ в точке $\left(x_{1}^{0}, x_{2}^{0}, x_{3}^{0}\right)$, то можно вычислить приращение скорости:

$$
\delta^{\prime} V_{j}=V_{j}+V_{j}^{\prime}-V_{j}^{0}
$$

С другой стороны приращение скорости равно сумме её среднего приращения (равного $V_{j}-V_{j}^{0}$ ) и пульсации приращения: 


$$
\delta^{\prime} V_{j}=\Delta V_{j}+\Delta^{\prime} V_{j}
$$

Сравнивая эту формулу с предыдущей, получаем, что пульсация приращения скорости равна пульсации скорости:

$$
\Delta^{\prime} V_{j}=V_{j}^{\prime}
$$

Запишем тензор:

$$
I_{j k}=\left(V_{j}^{\prime}-\Delta^{\prime} V_{j}\right) \cdot\left(V_{k}^{\prime}-\Delta^{\prime} V_{k}\right)
$$

Этот тензор должен быть нулевым, так как по предыдущему каждый сомножитель равен нулю. Производя перемножение, получим:

$$
\overline{-V_{j}^{\prime} V_{k}^{\prime}}=-\overline{V_{k}^{\prime} \Delta^{\prime} V_{j}}-\overline{V_{j}^{\prime} \Delta^{\prime} V_{k}}+\overline{\Delta^{\prime} V_{j} \Delta^{\prime} V_{k}}-I_{k}
$$

Мы можем вообразить два варианта наблюдения турбулентности в потоке. В первом варианте наблюдатель находится в какой либо постоянной точке потока, например с координатами $\left(x_{p}\right.$, $\left.x_{2}, x_{3}\right)$, и измеряет пульсации скорости в этой точке во времени. Это обычный способ экспериментальных измерений. Во втором варианте наблюдатель все время старается находиться в той точке потока, где скорость в данный момент времени совпадает со средней в точке $\left(x_{p}, x_{2}, x_{3}\right)$. Тогда наблюдатель будет вынужден перемещаться из одной точки потока в другую и вместо измерения пульсаций скорости будет измерять пульсацию координат.

Так же как и для пульсаций скорости для пульсаций координат мы можем ввести соответствующие обозначения:

$x_{j}$ - совокупность осредненных значений координат, $x_{j}^{\prime}$-пульсация координат, $\Delta^{\prime} x_{j}-$ пульсация приращения координат.

Для этих величин можно записать выражения, аналогичные (8), (9) и (10), так что получим:

$$
\Delta^{\prime} x_{j}=x_{j}^{\prime}
$$

Bсе осредненные величины пульсаций скорости и координат и пульсаций их приращений по определению равны нулю.

Очевидно, что оба способа наблюдений должны давать одинаковые результаты, и следовательно математические соотношения для интегральных параметров течения, базирующиеся на значениях пульсаций скорости и её приращения можно выразить через пульсацию координат и их приращений. Следует указать также, что по определению физическая средняя скорость есть

$$
\lim _{\tau \rightarrow 0} \frac{\Delta x_{j}}{\tau}=V_{j}
$$

Равным образом можно определить и пульсацию приращения скорости

$$
\lim _{\tau \rightarrow 0} \frac{\Delta^{\prime} x_{j}}{\tau}=\Delta^{\prime} V_{j}
$$

Кроме того, векторы пульсаций скорости и координат, а также векторы пульсаций их приращений - коллинеарны и противоположны по направлению [19].

Турбулентность как явление имеет стохастическую природу, она обусловлена случайными перемещениями малых, но имеющих конечные размеры, объемов жидкости, сохраняющих индивидуальность за время своего существования Т. В силу этого для описания некоторых процессов, связанных с явлением турбулентности, например, процессов переноса массы и импульса, пригоден аппарат статистической физики и механики $[23,24]$. Вeроятность обнаружения некоторого состояния механической системы, в которой протекают случайные процессы, возникающего из предыдущего состояния за время $\tau<$ Т описывается интегральным соотношением Смолуховского [25]. Указанное соотношение содержит функцию вероятности перехода системы из предыдущего состояния в последующее.

Доказано, что, если существуют предел (14), характеризующий интенсивность систематического процесса, а также предел:

$$
B_{j k}=\lim _{\tau \rightarrow 0} \frac{\overline{\Delta \xi_{j} \Delta \xi_{k}}}{\tau}
$$

характеризующий интенсивность стохастического процесса, то переходная вероятность имеет вид нормального распределения [8]. Параметром нормального распределения является тензор $\mathrm{B}_{\mathrm{jk}}$. В формуле (16) величины $\xi_{\mathrm{j}}$, $\xi_{\mathrm{k}}$ есть совокупность динамических переменных; для гамильтоновой системы - это набор импульсов и координат. Если рассматривать проблему в пространстве координат, то стоящее в верхней части дроби в формуле (16) осредненное произведение случайных изменений динамических переменных, есть осредненное произведение случайных перемещений. С другой стороны, теория случайных блужданий [23-25] для аналогичной переходной вероятности 
приводит к функции нормального распределения, параметром которой является матрица единичных перемещений, которая имеет вид:

$$
L_{j k}=\overline{l_{j}^{\prime} l_{k}^{\prime}}
$$

Выражения для переходной вероятности, полученные из интегрального соотношения Смолуховского в постранстве координат и из теории случайных блужданий совпадают, если положить:

$$
B_{j k}=\frac{\overline{l_{j}^{\prime} l_{k}^{\prime}}}{2} \cdot m
$$

где $m$ - число случайных перемещений за единицу времени.

Величина $m$ является скаляром, совпадающим с частотой пульсаций или перемещений. Коллинеарность пульсаций векторов длины (амплитуды) и скорости может быть показана путем следующих простых соображений.

Выделим в турбулентном потоке некоторую площадку $\mathrm{S}_{\mathrm{j}}$. Все пульсаии в объёме $\omega=S_{j} l_{j}^{\prime}$ достигнут площадки. С другой стороны этот объём равен $\omega=-S_{j} V_{j}^{\prime} \tau$. Знак “-“" поставлен потому, что векторы площадки и пульсации скорости имеют противоположные направления. Приравнивая оба последних выражения, получим:

$$
m=\frac{1}{\tau}=-\frac{V_{j}^{\prime}}{l_{j}^{\prime}}
$$

В выражениях для объёма и в формуле (19) по немым индексам суммирование не производится. Полагая, что применительно к турбулентному течению мерой интенсивности стохастического процесса являются коэффициенты турбулентной вязкости, можем записать:

$$
B_{j k}=\frac{1}{2} \varepsilon_{j k}
$$

Таким образом, мы показали, что выражения для коэффициентов турбулентной вязкости и диффузии являюся математическим тензором. Кроме того выражение для турбулентной вязкости в форме (18) в явном виде содержит частоту пульсаций.

Теперь положим, что выполняются следующие соотношения:

$$
\begin{aligned}
& \Delta^{\prime} V_{k}=M_{k i} \cdot \Delta^{\prime} x_{i} \\
& \Delta^{\prime} V_{j}=M_{j h} \cdot \Delta^{\prime} x_{h},
\end{aligned}
$$

где $M_{m n}-$ некий тензор, который предстоит отыскать.

Подставив выражения (21) и (22) в формулу (12), получим:

$$
\begin{aligned}
& -\overline{V_{j}^{\prime} V_{k}^{\prime}}=-M_{j h} \overline{\Delta^{\prime} x_{h} V_{k}^{\prime}}-M_{k i} \overline{\Delta^{\prime} x_{i} V_{j}^{\prime}}+ \\
& +M_{j h} M_{k i} \overline{\Delta^{\prime} x_{h} \Delta^{\prime} x_{i}}+I_{j k}
\end{aligned}
$$

Если пульсации приращения координат придать смысл перемещений, то есть обозначить:

$$
\Delta^{\prime} x_{h}=l_{h}^{\prime} ; \quad \Delta^{\prime} x_{i}=l_{i}^{\prime}
$$

то можно записать:

$$
-\overline{V_{j}^{\prime} V_{k}^{\prime}}=-M_{j h} \overline{l_{h}^{\prime} V_{k}^{\prime}}-M_{k i} \overline{l_{i}^{\prime} V_{j}^{\prime}}+M_{j h} M_{k i} \overline{l_{h}^{\prime} l_{i}^{\prime}}+I_{j k}
$$

Согласно правилу замены немых индексов из (25) вытекает:

$$
-\overline{V_{j}^{\prime} V_{k}^{\prime}}=-2 M_{j h} \overline{l_{h}^{\prime} V_{k}^{\prime}}+M_{j h} M_{k i} \overline{l_{h}^{\prime} l_{i}^{\prime}}+I_{j k}
$$

Исходя из принципа «независимости материала от системы отсчета» - принципа инвариантности в отношении преобразования координат Слеттери [16] указывает, что общий вид тензора осредненных турбулентных напряжений должен отображаться следующей формулой:

$$
-\bar{V}_{j} V_{k}=v_{0} I_{j k}+v_{1} S_{j k}+v_{2} S_{j i} S_{i k}
$$

Как мы видим обе формулы, (26) и (27), имеют одинаковую структуру.

Из соотношений (10), (21) и (24) непосредственно вытекает тензорная форма определения пульсации скорости:

$$
V_{k}^{\prime}=l_{i}^{\prime} M_{i k}
$$

Опуская в (19) нуль-тензор и учитывая последнее выражение, получим:

$$
-\overline{V_{j}^{\prime} V_{k}^{\prime}}=-l_{h}^{\prime} l^{\prime} M_{j h} M_{k i}=-\overline{l_{h}^{\prime} V_{k}^{\prime}} M_{j h}
$$

Тензор $l_{h}^{\prime} V_{k}^{\prime}$-всегда отрицательный, так как положительные пульсации скорости всегда сцеплены с отрицательными приращениями координат и наоборот [19].

Формулу (29) можно рассматривать как тензорное выражение пульсационных напряжений в терминах теории пути смешения. Она, следовательно, является тензорным обобщением формулы турбулентных напряжений, полученной Л. Прандтлем, а также гипотезы Буссинеска. 
Тензор:

$$
\varepsilon_{h k}=-M_{k i} \overline{l_{h}^{\prime} l_{i}^{\prime}}=-\overline{l_{h}^{\prime} V_{k}^{\prime}}
$$

представляет кажущуюся кинематическую турбулентную вязкость. Теперь задача заключается в том, чтобы выразить тензор $M_{j h}$ с помощью параметров, обычно измеряемых при исследовании турбулентных течений в каналах.

Сопоставим девиаторы тензоров:

$$
\begin{aligned}
& D_{j k}=\frac{\partial V_{j}}{\partial x_{k}} \\
& \text { и } \\
& M_{j k}=\frac{\Delta^{\prime} V_{j}}{\Delta^{\prime} x_{k}}
\end{aligned}
$$

По определению девиаторы указанных тензоров выражаются формулами:

$$
\begin{aligned}
& d_{j k}=D_{j k}-\frac{\delta_{j k}}{N} I_{D} \\
& m_{j k}=M_{j k}-\frac{\delta_{j k}}{N} I_{M}
\end{aligned}
$$

здесь $N$ - число координат.

Линейные инварианты тензоров (31) и (32) есть суммы диагональных компонент, т.е.:

$$
\begin{aligned}
& I_{D}=\frac{\partial V_{1}}{\partial x_{1}}+\frac{\partial V_{2}}{\partial x_{2}}+\ldots .+\frac{\partial V_{N}}{\partial x_{N}} \\
& I_{M}=\frac{\Delta^{\prime} V_{1}}{\Delta^{\prime} x_{1}}+\frac{\Delta^{\prime} V_{2}}{\Delta^{\prime} x_{2}}+\ldots . .+\frac{\Delta^{\prime} V_{N}}{\Delta^{\prime} x_{N}}
\end{aligned}
$$

С учетом выражений (19) и (24):

$$
I_{M}=-m N
$$

Для несжимаемой жидкости:

$$
I_{D}=0
$$

Положим, что:

$$
\beta d_{j k}=m_{j k}
$$

Тогда на основании (21), (22), (33) и (34) получим:

$$
M_{j k}=\beta D_{j k}-\frac{\delta_{j k}}{N}\left(\beta I_{D}-I_{M}\right)
$$

Для несжимаемой жидкости:

$$
M_{j k}=\beta D_{j k}-m \delta_{j k}
$$

Используя произведенные выкладки, запишем результирующее выражение для тензора турбулентных напряжений в несжимаемой жидкости:

$$
-\overline{V_{j}^{\prime} V_{k}^{\prime}}=-\overline{l_{h}^{\prime} l_{i}^{\prime}}\left(\beta D_{j h}-m \delta_{j h}\right)\left(\beta D_{k i}-m \delta_{k i}\right)
$$

Чтобы проиллюстрировать полученный результат, приведем выражения для объёмных и касательных напряжений при двухмерном течении несжимаемой жидкости, вытекающие из формулы (41). Для такого течения имеются многочисленные экспериментальные данные и расчеты по теориям Прандтля, Тейлора и Кармана. В частности в книге Г. Шлихтинга [19, стр. 524], помещены экспериментальные данные Г. Райхардта, который измерял турбулентные пульсации при течении воздуха в канале прямоугольного поперечного сечения.

Итак, для двухмерного течения $(N=2)$ из (42) следует:

$$
\begin{aligned}
& -\left(\overline{V_{1}^{\prime 2}}\right)=m^{2} \overline{l_{1}^{\prime 2}}-2 \beta m \overline{l_{1}^{\prime} l_{2}^{\prime}} \frac{\partial V_{1}}{\partial x_{2}} \\
& -\left(\overline{V_{2}^{\prime 2}}\right)=m^{2} \overline{l_{2}^{\prime 2}} \\
& -\left(\overline{V_{1}^{\prime} V_{2}^{\prime}}\right)=m^{2} \overline{l_{1}^{\prime} l_{2}^{\prime}}-\beta m \overline{l_{2}^{2}} \frac{\partial V_{1}}{\partial x_{2}}
\end{aligned}
$$

Эксперименты Г. Райхардта показали, что:

- Поперечная пульсация $\sqrt{\overline{V_{2}^{\prime 2}}}$ мало изменяется по поперечной координате и не зависит от поперечного градиента осевой скорости. То же следует из формулы (44).

- На осевой плоскости канала, где производная от осевой скорости по поперечной координате равна нулю $\left(\frac{\partial V_{1}}{\partial x_{2}}=0\right)$, а поперечная $\sqrt{\overline{V_{2}^{\prime 2}}}$ и продольная $\sqrt{\overline{V_{1}^{\prime 2}}}$ пульсации отличаются друг от друга только примерно на $10 \%$, что следует из формул (43) и (45). 
- Продольная пульсация связана с производной от скорости течения по поперечной координате и вблизи от стенки канала, где эта производная велика, имеет явно выраженный максимум, что следует из формулы (43).

- Касательные турбулентные напряжения - $\rho\left(\overline{V_{1}^{\prime} V_{2}^{\prime}}\right)$ на осевой плоскости канала равны нулю и возрастают к стенке канала по мере роста модуля поперечной производной от осевой скорости, что следует из формулы (45).

Видно, что формулы (43)-(45) качественно полностью соответствуют экспериментальным данным Г. Райхардта. Следует заметить, что на осевой плоскости канала компоненты тензора перемещений $\bar{l}_{j} l_{k}$ только при $j \neq k$ равны нулю по условию симметрии потока относительно упомянутой плоскости. Известно [19], что гипотеза Прандтля подвергалась неоднократным модификациям, так как из неё следовало, что в точках экстремума скорости турбулентные пульсации исчезают. Однако эксперименты этого не подтверждают. Формулы (43)-(45) свободны от этого противоречия.

\section{Выводы}

Таким образом, мы получили тензорную форму гипотезы Буссинеска, которая может быть использована при преобразованиях уравнения Рейнольдса и, как нам представляется, дает трактовку кажущейся турбулентной вязкости как тензорной величины.

\section{Условные обозначения:}

$B_{j k}-$ коэффициент интенсивности стохастического процесса, $\mathrm{M}^{2} /$ сек;

$D_{j k}-$ тензор, характеризующий поле скоростей осредненного течения, 1/сек;

$d_{j k}$ - девиатор тензора $D_{j k}, 1 /$ сек;

$I_{j k}$ - нуль-тензор;

$I_{D}$ - линейный инградиент тензора $D_{j k}, 1 /$ сек;

$I_{M}$ - линейный инградиент тензора $M_{j k}, 1 /$ сек;

$L_{j k}$ - тензор перемещений, м $^{2}$;

$l_{j}^{\prime}, l_{k}^{\prime}-$ компоненты вектора пульсационных перемещений, м;

$l$ - длина «пути перемешивания», м;

$M_{j k}$ - тензор, характеризующий поле пульсационных скоростей, 1/сек;

$m_{j k}$ девиатор тензора $M_{j k}, 1 /$ сек;

$m$ - частота пульсаций, $1 /$ сек;

$N$ - число координат;

$P$ - давление, кг $/ \mathrm{M}^{2}$;

$S_{j k}$ - тензор скоростей деформаций, $1 /$ сек;

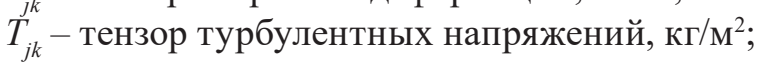

$u$ - осредненная скорость течения в плоском канале, м/сек;

$V_{p} V_{k}-$ компоненты вектора осредненной скорости течения, м/сек;

$V_{j}^{\prime}, V_{k}^{\prime}-$ компоненты вектора пульсационной скорости течения, м/сек;

$\Delta^{\prime} V_{j} \Delta^{\prime} V_{k}$ - компоненты вектора пульсаций приращения осредненной скорости течения, м/сек;

$\delta^{\prime} V_{j}$ компоненты вектора приращения истинной скорости течения, м/сек;

$x_{j} x_{k}-$ компоненты координат, м;

$x_{j}, x_{k}{ }_{k}$ - пульсации координат, м;

$\Delta x x_{j}^{\prime} x_{k}-$ пульсации приращений координат, м;

$y$ - поперечная координата, м;

$\beta$ - коэффициент;

$\delta_{j k}-$ тензорная единица;

$\varepsilon_{j k}-$ тензор турбулентной кинематической вязкости, $\mathrm{M}^{2} /$ сек;

$\varepsilon$ - турбулентная кинематическая вязкость, $\mathrm{M}^{2} / \mathrm{ce \kappa ;}$

$T$ - время осреднения, сек;

$\tau_{j k}$ - тензор вязких напряжений, кг/м²;

$\tau$ - время, сек;

$\Delta^{\prime} \xi_{j} \Delta^{\prime} \xi_{k}-$ пульсации динамических переменных, м;

\section{Индексы:}

0 - начальное значение;

$i, j, k, h=1,2,3 \ldots N-$ номера компонент векторов или тензоров;

$m, n=i, j, k, h=1,2,3 \ldots N$ - номера компонент тензоров.

\section{Литература}

1. Белов И.А., Исаев С.И. Моделирование турбулентных течений. СПб: Балт.гос.техн. ун-т. 2001. 109 с.

2. Гарбарук А.В., Лапин Ю.В., Стрелеи М.Х. Теплофизика высоких температур. 2001. Т.39. №4 С. 589598.

3. Лабусов А.Н., Лапин Ю.В. Теплофизика высоких температур. 2000. Т. 38. № 3. С. 458-467.

4. Полежаев Ю.В., Коршунов А.В., Габбасова Г.В. Теплофизика высоких температур. 2007. Т. 45. №3. C. 378-383.

5. Рудаков О.А., Митрофанов В.А. Вестник Самарского государственного аэрокосмического университета. 2002. №2(2). С. 92-96.

6. Смирнов Е.М., Абрамов А.Г., Иванов Н.Г., Корсаков А.Б. Научно-технические ведомости (НТВ). 2004. №2. C. 33-47.

7. Трунев А.П. Научный журнал КубГАУ. 2016. № 118(04). C. 1-19.

8. Трунев А.П. Научный журнал КубГАУ. 2016. № 119(05). С. 1111-1133.

9. Трунев А.П. Научный журнал КубГАУ. 2016. № 124(10). C. 1-26. 
10. Шарфереи Б.П., Дмитриев С.П. Научное приборостроение. 2018. Т. 28. №3. С. 101-108.

11. Смирнов E.M., Гарбарук A.B. Течения вязкой жидкости и модели турбулентности: методы расчета турбулентных течений. СПб: Санкт-Петербургский государственный политехнический университет. 2010. 139 c.

12. Коренев Г.В. Тензорное исчисление. М.: Изд-во МФТИ. 2000. 240 с.

13. Жилин П.А. Векторы и тензоры второго ранга в трехмерных пространствах. СПб.: Нестор. 2001. 275 с.

14. Денисова И.П. Введение в тензорное исчисление и его приложения. М.: Изд. УНЦ ДО. 2004. 230 с.

15. Дмитриенко Ю.И. Тензорное исчисление. М.:Высшая школа. 2001.575 с.

16. Лойцянский Л.Г. Механика жидкости и газа. М.: Дрофа. 2003. 840 с.

17. Слеттери Дж.С. Теория переноса импульса, энергии и массы в сплошных средах. М.: Энергия. 2001. $448 \mathrm{c}$.
18. Прандтль Л. Гидроаэромеханика.М.- Ижевск: НИЦ «Регулярная и хаотическая динамика». 2000. 576 с.

19. Шлихтинг Г. Теория пограничного слоя. М.: Наука. 1969. $744 \mathrm{c}$.

20. Sergeev S.P., Krasnushkina N.V., Maidurov N.P., Petrovskaya M.A.. Catalysis in Industry. 2011. V. 3. No3. P. 283-289.

21. Сергеев С.П., Никифоров Ф.Ф., Афанасьев С.В.,. Шевченко Ю.Н. Изв. вузов. Химия и хим. технология. 2019. Т. 62. №4. С. 143-151.

22. Terril R.M. IMA, J. Appl. Math. 1984. V. 33. №2. P. 169-174.

23. Василевский А.С., Мултановский В.В. Статистическая физика и термодинамика. М.: Просвещение. 2005. $256 \mathrm{c}$

24. Щеголев И.Ф. Элементы статистической механики, термодинамики и кинетики. Долгопрудный: Интеллект. 2008. 208 с.

25. Галкин В.A. Уравнение Смолуховского. М.: ФИЗМАТЛИТ. 2001. 336 с. 